\title{
Onset And Burden of Lower Limb Lymphedema After Radical Prostatectomy: A Cross-Sectional Study
}

\section{Manuel Neuberger ( $\nabla$ manu.neuberger@gmail.com )}

Department of Urology and Urological Surgery, University Medical Centre Mannheim https://orcid.org/0000-0003-4573-5134

\section{Laura Schmidt}

Institute of Psychology, University of Heidelberg

\section{Frederik Wessels}

Department of Urology and Urological Surgery, University Medical Centre Mannheim

\section{Miriam Linke}

Department of Dermatology, Venereology and Allergology, University Medical Centre Mannheim

\section{Carina Müller}

Physiotherapy, Service Center Therapy, University Medical Centre Mannheim

\section{Niklas Westhoff}

Department of Urology and Urological Surgery, University Medical Centre Mannheim

\section{Philipp Nuhn}

Department of Urology and Urological Surgery, University Medical Centre Mannheim Jost von Hardenberg

Department of Urology and Urological Surgery, University Medical Centre Mannheim

\section{Research Article}

Keywords: Prostate cancer, Lower Extremity, Manual Lymphatic Drainage, Surveys and Questionnaires, Lymph Node Excision, Postoperative Complications

Posted Date: July 14th, 2021

DOl: https://doi.org/10.21203/rs.3.rs-620768/v1

License: (c) (i) This work is licensed under a Creative Commons Attribution 4.0 International License. Read Full License

Version of Record: A version of this preprint was published at Supportive Care in Cancer on September 3rd, 2021. See the published version at https://doi.org/10.1007/s00520-021-06520-2. 


\section{Abstract}

\section{Objectives}

To explore men's onset and burden of lower limb lymphedema (LLL) after radical prostatectomy (RP) with pelvic lymph node dissection.

\section{Patients and methods}

A cross-sectional survey-based study was conducted nation-wide and web-based in Germany. Part 1 included 15 multidisciplinary compiled questions with three questions from Short Form 12 Health Survey (SF-12) and WHO activity recommendation, part 2 the validated German Lymph-ICF-Questionnaire (Lymph-ICF-LL). Subgroup comparisons and simple regression analyses were used to identify factors associated with therapy and burden of LLL, followed by multiple regression analyses to explain variance in impairment in the patients' daily life.

\section{Results}

54 patients completed the survey. Median time of LLL-onset was reported with 2.0 (0.5-9.75) months after RP. 19 patients (35.2\%) reported bilateral lymphedema, 28 (51.9\%) the use of individually fitted compression stockings (CS), 25 (46.3\%) of manual lymphatic drainage (LD) and 26 (48.1\%) complete regression.

The Lymph-ICF-LL revealed a higher total burden for patients with an active LLL compared to complete regression (total score: 25.5 vs. $11.9, p=0.01)$ specially for "physical function" ( 28.3 vs. $12.9, p=0.004)$ and "mental function" (26.2 vs. 6.7, $p<0.001)$. In multiple linear regression analysis, a higher BMI $(\beta=0.28)$, lower subjective general health $(\beta=-0.48)$ and active lymphedema $(\beta=0.28)$ were significant predictors of higher reported impairments in the Lymph-ICF-LL, accounting for $45.4 \%$ of variance.

\section{Conclusion}

Men with LLL after RP with PLND report a significant burden in daily life. Bbasic therapy needs to be offered early. Postoperative onset of LLL is variable, which should be considered when assessing complications after RP.

\section{Introduction}

Most prostate cancers are detected in a localized stage [1]. One of the widely recommended and chosen treatment options represents the radical prostatectomy (RP; open, robotic-assisted laparoscopic or conventional laparoscopic) [2]. Nearly nine out of ten RPs are accompanied by pelvic lymph node dissection (PLND) with a median yield of 19 lymph nodes in intermediate or high-risk prostate cancer [3]. While PLND is important for correct staging of lymph node status, lower limb lymphedema (LLL) can be one of its side-effects. 
LLL can have a significant impact on quality of life [4]. However, lymphedema in general, including LLL linked to prostate cancer, is described as "under-recognized and under-treated"[5]. Reasons for this could lie in the recommendation to capture non-oncological outcome after RP with PLND. Two methods are suggested by the international consortium of health outcome measures (ICHOM) [6]: 1. Clavien-Dindo classification for grade $\geq 3$ complications within the first 6 months and 2. Patient-reported outcomes (PROMs) using the EPIC-26 questionnaire. The questionnaire covers only four domains (urinary irritative/obstructive, bowel, sexual and hormonal). Even recent large prospective multicenter trials assessing radical prostatectomy focused only on EPIC-26 and thereby did not capture LLL [7]. In a large RP series (PLND in $75 \%$ of patients), with a standardized reporting of complications only within 30 days postoperatively, LLL was reported as Clavien-Dindo grade 1 and made up a small proportion $(0.7 \%)$ of all registered complications [8]. As it is classified as Clavien-Dindo grade $<3$, LLL is not systematically captured following the international recommendations for patient-centered outcomes and thus data regarding LLL is sparse.

Due to the long natural course of prostate cancer especially potential long-term complication with relevant impact on patient's quality of life should be longitudinally assessed.

This study aims to obtain a differentiated characterization of the onset and burden of LLL after RP as well as the relation and overlap of the use of individually fitted compression stockings (CS) and manual lymphatic drainage (LD). Furthermore, using the validated version of the German Lymph-ICF-Lower Limb (Lymph-ICF-LL), we want to provide new insights on how and if LLL leads to an impairment in different domains of the daily life and which factors are associated with higher impairments.

\section{Patients And Methods}

\section{Recruitment}

The questionnaire was designed as a web-based cross-sectional survey using SoSci Survey (SoSci Survey GmbH, Munich, Germany, https://www.soscisurvey.de/) and thereafter distributed via the German Federal Association Prostate Cancer Self-Help e.V. (Bundesverband Prostatakrebs Selbsthilfe e.V.) e-mail distribution list. Men with active or regression of LLL after RP were invited to participate. Data were collected online in March and April 2021. Ethical approval was obtained from the University of Heidelberg's Ethics Committee II (Medical Faculty Mannheim, reference number 2021 - 517).

\section{Questionnaire}

The questionnaire was created in an interdisciplinary collaboration including colleagues from the fields of psychology, dermatology and physiotherapy. After providing informed consent, its 43 questions collected demographic and physical health data (items $1-3$ of the Short Form 12 Health Survey (SF-12) [9]), physical activity (WHO activity recommendation [10]), details on RP, PLND and the postoperative course (e.g. diagnosis of metastases, radiotherapy) and the treatment of LLL and its possible complications (e.g. 
erysipelas). These questions provided answers as (dropdown) selections, thus preventing inadvertent mis-entries.

The questionnaire was supplemented by the validated German version of the Lymphedema Functioning, Disability and Health Questionnaire for Lower Limb Lymphedema (Lymph-ICF-LL) to assess quality of life, which consists of 28 questions regarding impairments in five domains: physical function, mental function, general tasks/household activities, mobility activities, and life domains/social life [11]. Each question uses a 11-point Likert scale from 0 to 10. Higher scores indicate stronger impairments. The Lymph-ICF-LL is considered the first reliable and valid questionnaire for patients with LLL in German [12]. For the evaluation of the Lymph-ICF-LL we followed Devoogdt et al.'s instructions [11]:

\section{$\frac{\text { Sum of scores on questions }}{\text { Total number of answered questions }} \times 10$}

. Scores for each of the 5 domains were determined in the same way.

Results for the total score and the 5 domains range from 0 to 100. It is recommended by ICF taxonomy to interpret scores as follows: 0-4 = no problem, 5-24 small problem, 25-49 moderate problem, 50-95 severe problem, $96-100$ very severe problem [11]. Patients spent on average 16.6 minutes $( \pm 5.9)$ completing the survey.

\section{Statistical Analysis}

Frequencies and proportions were assessed for categorical variables, while means, medians, and interquartile ranges (IQR) were computed for continuous variables. Comparisons between subgroups were performed using t-tests for interval scaled variables and $\chi^{2}$-test for nominal scaled variables. Significant results in bivariate analyses were further analyzed in multiple linear and logistic regression analyses. All tests were two-sided with a statistical significance set at $p<0.05$. Analyses were performed using JMP®15 (SAS Institute Inc., Cary, North Carolina, USA).

\section{Results}

The survey link was opened 400 times and 158 datasets were created. Subsequently, 104 of these responses were removed prior to analysis due to incomplete responses. The remaining 54 complete datasets were included into statistical analysis. Table 1 summarizes the baseline characteristics of responding patients.

Table 1: Baseline characteristics of study cohort. 


\begin{tabular}{|c|c|}
\hline \multirow[t]{2}{*}{ Characteristic } & No. (\% or SD) \\
\hline & Cohort $(n=54)$ \\
\hline \multicolumn{2}{|l|}{ Age [years] } \\
\hline - At RP, mean (SD) & $65.0(6.8)$ \\
\hline - At survey, mean (SD) & $74.4(6.6)$ \\
\hline \multicolumn{2}{|l|}{$\mathrm{BMI}, \mathrm{kg} / \mathrm{m}^{2}$} \\
\hline - Mean (SD) & $26.5(3.5)$ \\
\hline Diabetes mellitus $(\mathrm{n}, \%)$ & $10(18.5)$ \\
\hline Moderate physical activity $(\mathrm{n}, \%)^{1}$ & $38(70.4)$ \\
\hline \multicolumn{2}{|l|}{ Date Difference RP- Questionnaire [years\} } \\
\hline - Mean (SD) & $7.4(5.8)$ \\
\hline \multicolumn{2}{|l|}{ Surgical approach } \\
\hline - Open RP (n, \%) & $22(40.7)$ \\
\hline - Laparoscopic RP (n, \%) & $5(9.3)$ \\
\hline - Robotic-assisted, laparoscopic RP (n, \%) & $27(50.0)$ \\
\hline Pelvic lymph node dissection $(n, \%)^{\dagger}$ & $49(96.1)$ \\
\hline \multicolumn{2}{|l|}{ No. of dissected lymph nodes ${ }^{\dagger+}$} \\
\hline - Mean (SD) & $16.0(11.4)$ \\
\hline Postoperative Radiotherapy (n, \%) & $20(37.0)$ \\
\hline \multicolumn{2}{|l|}{ Date Difference RP- Radiotherapy [months] ${ }^{\dagger+\dagger}$} \\
\hline - Mean (SD) & $29.2(47.1)$ \\
\hline Postoperative ADT (n, \%) & $21(38.9)$ \\
\hline \multicolumn{2}{|l|}{ Duration of ADT [months] ${ }^{\mathbb{}}$} \\
\hline - Mean (SD) & $35.6(26.1)$ \\
\hline Abdominal or pelvic metastasis after RP $(n, \%)$ & $16(30.0)$ \\
\hline \multicolumn{2}{|c|}{ Date Difference RP - abdominal or pelvic metastasis, months } \\
\hline - Mean (SD) & $41.4(44.5)$ \\
\hline Unilateral LLL & $35(64.8)$ \\
\hline - Complete remission (n, \%) & $16(45.7)$ \\
\hline
\end{tabular}


- Partial remission ( $n, \%)$

$8(22.9)$

- No changes $(n, \%)$

Bilateral LLL

19 (35.2)

- Complete remission ( $\mathrm{n}, \%)$

$10(52.6)$

- Partial remission ( $n, \%)$

$5(26.3)$

- No changes $(n, \%)$

$4(21.1)$

Date Difference RP - LLL [months]

- Mean (IQR)

$8.2(0.5-9.8)$

Complete regression of LLL $(\mathrm{n}, \%)$

$26(48.1)$

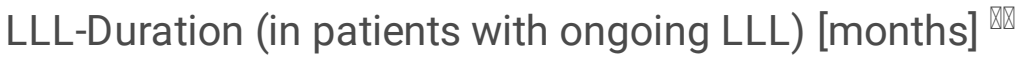

- Mean (SD)

$70.1(56.9)$

Compression stockings $(\mathrm{n}, \%)$

$28(51.9)$

Manual lymphatic drainage $(n, \%)$

$25(46.3)$

Erysipela (n, \%)

2 (3.7)

SF12-Item 1

General Health perception*

- Mean (SD)

$2.89(0.79)$

SF12-Item 2

Limitations in moderate activities during a typical day

- Severely limited (\%)

$4(7.4)$

- Somewhat limited (\%)

$27(50.0)$

- Not restricted at all (\%)

$23(42.6)$

SF12-Item 3

Limitations in climbing several flights of stairs during a typical day.

- severely limited (\%)

- somewhat limited (\%)

- not restricted at all (\%)

$24(44.4)$

Physical activity per week [minutes] ${ }^{2}$

- Mean (SD)

$220.2(262.8)$ 
*coded 1-5 (higher scores indicate higher subjective health), 1patients who follow the WHO recommendations of at least 150 minutes of moderate physical activity per week, 2 based on the data from the 38 patients meeting the above-mentioned WHO recommendations regarding moderate physical activity, tdata of 3 patients missing, + tata of 5 patients missing, +++ based on data of the 20 patients

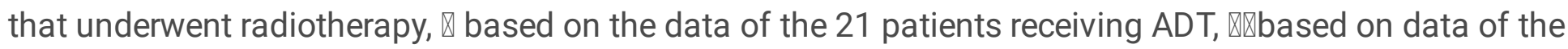
28 patients with an active LLL at the time of survey

At the time of the survey, LLL was still present in $n=28$ men (51.9\%), whereas the remaining patients reported a complete regression. Most common reported LLL-onset was within the first month postoperatively $(n=21(38.9 \%)) .61 \%(n=33)$ of LLL were reported with an onset within 3 months, $70.3 \%$ $(n=38)$ within 6 months and 81.4\% $(n=44)$ within the first year after RP. $14.0 \%(n=8)$ of LLLs were reported with an onset $>36$ months after RP $(n=8,14 \%)$. We defined a group of "late onset" $L L L$, containing the $28.6 \%$ of patients with LLL-onset $>12$ months after RP. This group showed a higher percentage of abdominal metastasis ( $50 \%$ vs. $25 \%$ ) and received RT more often ( $50 \%$ vs. $34 \%$ ), however both findings did not show statistical significance $(p=0.13$ and $p=0.35$, respectively). Figure $1 \mathrm{~A}$ shows the onset of LLL after RP as a reverse Kaplan-Meier curve.

Figure 1B shows the relation and overlap of patients in need of individually fitted compression stockings (CS) and manual lymphatic drainage (LD). The temporal relationship between the appearance of LLL and the beginning of the use of CS and LD is shown in Fig. 1C.

$28(52.9 \%)$ patients used CS. The mean time between LLL-onset to CS was reported with 3.78 months ( \pm 9.18). Of the 28 patients, who use or used CS, 21 patients $(75.0 \%)$ reported the frequency of stocking usage after fitting of CS: mostly used on a daily basis $(n=13(59.1 \%))$, four patients $(9.5 \%)$ reported the use on three to five days a week and four patients $(9.5 \%)$ on one or two days per week. Of the 28 patients with an active LLL, 15 (53.6\%) reported the use of CS within four weeks prior to the survey.

LD was reported by 25 patients (46.3\%) with a start 4.1 months $( \pm 7.13)$ after LLL-onset. LD-frequency was reported with $1 x /$ week most commonly $(n=13(52 \%))$, less than once a week in three $(12.0 \%)$ and more than once a week in $9(36.0 \%)$ cases. 14 (56.0\%) of the 25 patients, who reported need of LD, stated an ongoing need of LD, all of which also reported active LLL. In two cases, frequency could be decreased since LD-onset. Both reductions were reported possible 24 months after the start of LD. Out of the 11 patients, in which LD could be terminated, 4 (36.4\%) required LD for more than 12 months. Two patients received LD for more than 36 months before it could be terminated.

Additional therapy besides CS and LD was reported by 4 patients $(7.4 \%)$ and contained medication, compression device and puncture.

First, pursuing the assumption that LLL is more severe, if manual LD is necessary, we analyzed which factors differ between those participants that received LD or not: Subjective general health (as assessed using the SF12 GH1), time between RP and LLL-onset, bilateral LLL, number of LN removed during PLND and usage of CS showed significant results. In multiple logistic regression analysis with LD (yes/no) as 
dependent variable, subjective general health, bilateral LLL and number of removed $L N$ were significant predictors and accounted for $46 \%$ of the variability. Results are shown in Table $2 \mathrm{~A}$.

Second, we analyzed possible factors regarding whether or not the LLL partially or completely regressed. In subgroup comparison (t-test / $\chi^{2}$ ) subjective general health, physical activity and time between RP and LLL-onset, receiving LD and radiotherapy after RP showed significance. In multiple logistic regression analysis with LLL-regression (no / yes) as dependent variable, none of these variables remained significant. Results are shown in Table 2B. 
Table 2

Group comparison and multiple logistic regression analysis of variables that could $\mathbf{A}$ explain the "need of LD" and B explain whether or not a LLL partially or completely regresses.

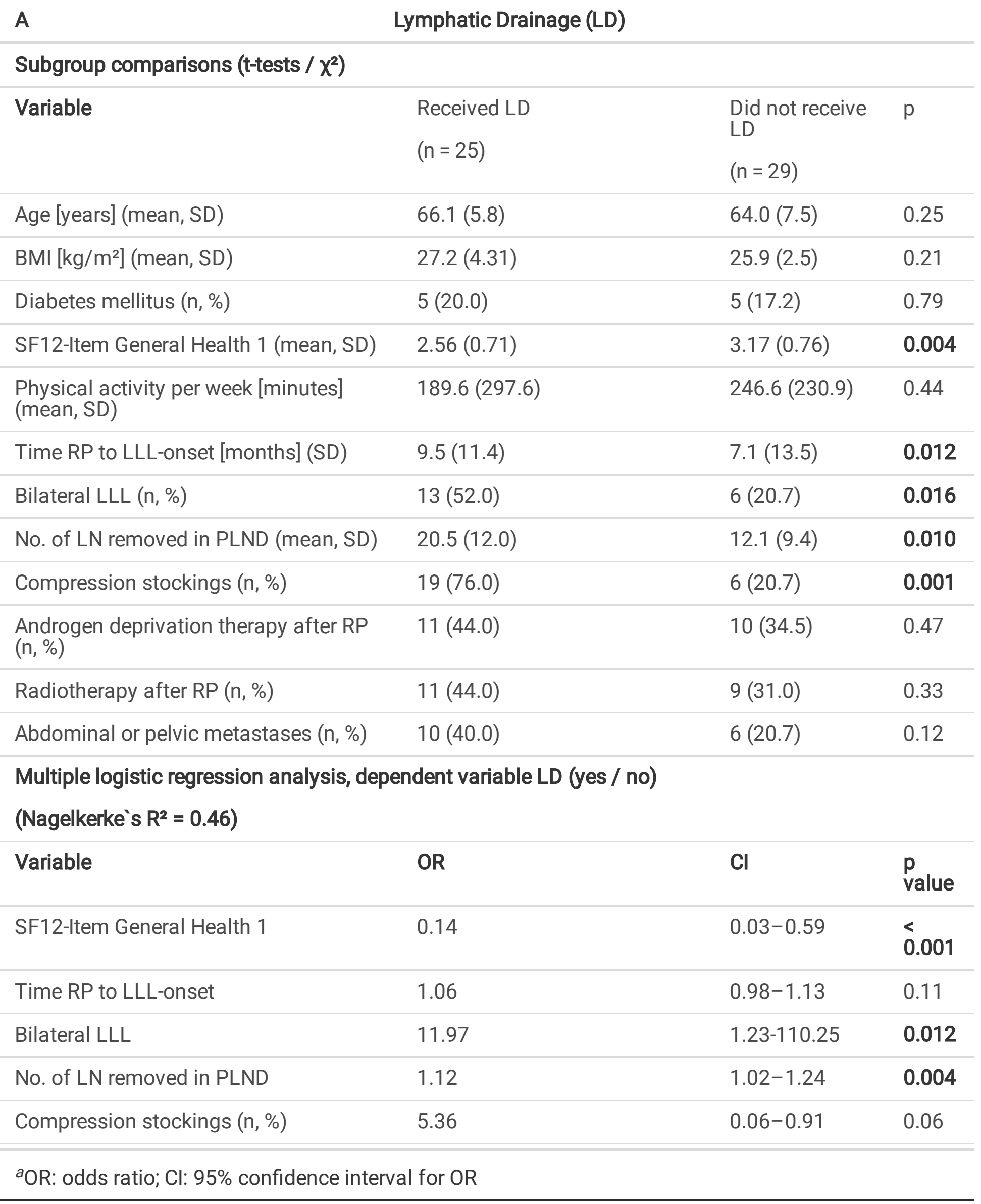




\begin{tabular}{|c|c|c|c|}
\hline \multicolumn{4}{|c|}{ Lymphatic Drainage (LD) } \\
\hline B & \multicolumn{3}{|c|}{ Regression of Lower Limb Lymphedema (LLL) } \\
\hline \multicolumn{4}{|l|}{ Subgroup comparisons (t-tests / $\chi^{2}$ ) } \\
\hline \multirow[t]{2}{*}{ Variable } & $\begin{array}{l}\text { LLL regression (partial or } \\
\text { complete) }\end{array}$ & $\begin{array}{l}\text { No LLL } \\
\text { regression }\end{array}$ & $\mathbf{p}$ \\
\hline & $(\mathrm{N}=39)$ & $(\mathrm{N}=15)$ & \\
\hline Age [years] (mean, SD) & $65.4(7.0)$ & $64.0(6.2)$ & 0.48 \\
\hline BMI [kg/m²] (mean, SD) & $26.0(3.1)$ & $28.1(4.2)$ & 0.10 \\
\hline Diabetes Mellitus (n, \%) & $7(18.0)$ & $3(20.0)$ & 0.86 \\
\hline SF12-Item General Health 1 (mean, SD) & $3.1(0.7)$ & $2.5(0.8)$ & 0.026 \\
\hline $\begin{array}{l}\text { Physical activity per week [minutes] } \\
\text { (mean, SD) }\end{array}$ & $272.8(289.0)$ & $83.3(83.5)$ & $\hat{0} .001$ \\
\hline Time RP to LLL-onset [months] (SD) & $5.8(11.0)$ & $14.4(14.4)$ & 0.049 \\
\hline Bilateral LLL (n, \%) & $15(38.5)$ & $4(26.7)$ & 0.42 \\
\hline PLND (n, \%) & $35(97.2)$ & $14(93.3)$ & 0.51 \\
\hline No. of LN removed in PLND (mean, SD) & $17.4(12.5)$ & $12.8(7.9)$ & 0.12 \\
\hline Compression stockings (n, \%) & $18(46.1)$ & $10(66.7)$ & 0.18 \\
\hline Manual lymphatic drainage $(\mathrm{n}, \%)$ & $14(35.9)$ & $11(73.3)$ & 0.014 \\
\hline $\begin{array}{l}\text { Androgen deprivation therapy after RP } \\
(n, \%)\end{array}$ & $13(33.3)$ & $8(53.3)$ & 0.18 \\
\hline Radiotherapy after RP (n, \%) & $10(25.6)$ & $10(66.7)$ & 0.005 \\
\hline Abdominal or pelvic metastases $(n, \%)$ & $10(25.6)$ & $6(40.0)$ & 0.30 \\
\hline
\end{tabular}

Multiple logistic regression analysis, dependent variable "complete or partial LLL regression" (yes / no) (Nagelkerke's $R^{2}=0.34$ )

\begin{tabular}{llll} 
Variable & OR & Cl & $\begin{array}{c}\text { P } \\
\text { value }\end{array}$ \\
\hline SF12-Item General Health 1 & 0.51 & $0.15-1.80$ & 0.28 \\
\hline Physical activity per week & 0.99 & $0.99-1.00$ & 0.08 \\
\hline Time RP to LLL-onset & 1.06 & $0.99-1.13$ & 0.05 \\
\hline Manual lymphatic drainage & 4.00 & $0.77-20.83$ & 0.09
\end{tabular}

${ }^{a} \mathrm{OR}$ : odds ratio; $\mathrm{Cl}$ : 95\% confidence interval for OR 


A Lymphatic Drainage (LD)

Radiotherapy after RP

3.01

$0.61-14.81$

0.17

${ }^{a}$ OR: odds ratio; $\mathrm{Cl}$ : 95\% confidence interval for OR

The Lymph-ICF-LL questionnaire showed significant differences in group comparisons for total score as well as in the sub-domains "physical function", "mental function" and "general tasks/household activities": Patients with an active LLL had total scores indicating a moderate problem (25-49) as defined by the total score as well as for physical and mental function, whereas the scores in the group with completely regressed LLLs indicated a small problem (5-24). Results are shown in Table 3A. Subgroup comparisons between patients with $(n=28)$ or without active LLL $(n=26)$ revealed that those with an active LLL received LD more often. Results are shown in Table 3B.

A higher BMI, a lower subjective health (SF-12) and the presence of an active LLL remained significant predictors of higher impairments reported in the Lymph-ICF-LL in a multiple linear regression analysis. Together, these predictors were able to explain $45.4 \%$ of variance in the Lymph-ICF-LL total score. Results are shown in Table $3 \mathrm{C}$. 
Table 3

A Results of the Lymph-ICF-LL comparing patients with an active LLL versus patients who have experienced LLL that has had completely regressed prior to the conduction of the survey. B Subgroup comparison between patients with an active LLL and a completely regressed LLL C Simple and multiple

linear regression in order to detect variables associated with the outcome of the Lymph-ICF-LL.

A

Lymph-ICF-LL Score (total score and sub-domains)

Subgroup comparisons (t-test)

\section{Variable}

Total (mean, SD) *

Physical function (mean, SD) *

Mental function (mean, SD) *

General tasks / Household activities (mean, $\mathrm{SD})$ *

Mobility (mean, SD) *

Life Domains /Social Life (mean, SD) *

B

Subgroup comparisons (t-test / $\chi^{2}$-test)

\section{Variable}

Age [years] (mean, SD)

BMI $\left[\mathrm{kg} / \mathrm{m}^{2}\right]$ (mean, SD)

Diabetes Mellitus ( $n, \%)$

SF12-Item General Health 1 (mean, SD)

Moderate physical activity per week [minutes]

Bilateral LLL $(\mathrm{n}, \%)$

No. of LN removed in PLND (mean, SD)

Compression stockings ( $\mathrm{n}, \%)$

Manual lymphatic drainage $(n, \%)$

Androgen deprivation therapy after RP (n, \%)

Radiotherapy after RP ( $n, \%)$

${ }^{\mathrm{a}} \mathrm{Adj} . \mathrm{R}^{2}=0.45$, *higher scores indicate stronger impairments, $\beta=$ beta coefficient

Lower Limb Lymphedema (LLL) active LLL

$(\mathrm{N}=28)$

$65.0(5.6)$

$27.0(4.5)$

$5(17.9)$

$2.8(0.9)$

$208.6(279.8)$

$9(32.4)$

$27.0(17.9)$

$17(60.7)$

18 (64.3)

13 (46.4)

$12(42.9)$

Page $12 / 20$ active LLL not active LLL $\quad$ p

$(\mathrm{N}=28) \quad(\mathrm{N}=26)$

$25.5(3.6)$

$11.9(3.7)$

0.010

$28.3(3.5)$

$12.9(3.6)$

0.004

$26.2(3.8)$

$6.7(4.0)$

$<0.001$

$18.3(4.2)$

$7.1(4.3)$

0.068

$28.3(4.6)$

$17.2(4.8)$

0.10

$21.2(4.3)$

$13.4(4.5)$

0.22 not active LLL p

$(\mathrm{N}=26)$

$65.0(7.9)$

1.0

$26.1(2.1)$

0.34

5 (19.2)

0.90

$3.0(0.7)$

0.18

232.7 (248.2)

0.74

10 (38.5)

0.63

13.7 (9.6)

0.19

11 (42.3)

0.18

7 (26.9)

0.005

8 (30.8)

0.24

8 (30.8)

0.36 


\begin{tabular}{|c|c|c|c|c|}
\hline A & \multicolumn{4}{|c|}{ Lymph-ICF-LL Score (total score and sub-domains) } \\
\hline Abdominal or pelvic metastases $(n, \%)$ & \multicolumn{2}{|c|}{$9(31.1)$} & $7(26.9)$ & 0.67 \\
\hline C & \multicolumn{4}{|c|}{ Predictors of Lymph-ICF-LL total score } \\
\hline \multirow[t]{2}{*}{ Variable } & \multicolumn{2}{|c|}{$\begin{array}{l}\text { simple linear } \\
\text { regression }\end{array}$} & \multicolumn{2}{|c|}{ multiple linear regression } \\
\hline & $\beta$ & $\mathbf{p}$ & $\beta$ & p \\
\hline Age [years] & -0.01 & 0.75 & & \\
\hline BMI $\left[\mathrm{kg} / \mathrm{m}^{2}\right]$ & 0.43 & 0.001 & 0.28 & 0.014 \\
\hline Diabetes Mellitus & -0.02 & 0.89 & & \\
\hline SF12-Item General Health 1 & -0.58 & $<0.001$ & -0.47 & $\begin{array}{l}<.001 \\
0.00\end{array}$ \\
\hline Moderate physical activity per week & -0.33 & 0.02 & -0.14 & 0.22 \\
\hline Time RP to LLL-onset & 0.20 & 0.15 & & \\
\hline Active LLL & 0.35 & 0.01 & 0.28 & 0.017 \\
\hline Bilateral LLL & -0.07 & 0.64 & & \\
\hline No. of LN removed in PLND & -0.12 & 0.41 & & \\
\hline Compression stockings & 0.19 & 0.17 & & \\
\hline Manual lymphatic drainage & 0.20 & 0.14 & -0.17 & 0.16 \\
\hline Androgen deprivation therapy after RP & 0.07 & 0.64 & & \\
\hline Radiotherapy after RP & 0.32 & 0.02 & 0.09 & 0.43 \\
\hline Abdominal or pelvic metastases & 0.18 & 0.19 & & \\
\hline
\end{tabular}

\section{Discussion}

This study aimed to describe the onset and burden of men with LLL after RP. Using the Lymph-ICF-LL for the first time in this specific group of patients, this questionnaire was able to detect differences between patients suffering from an active LLL and patients, whose LLL has regressed, especially regarding physical and mental skills related to LLL.

The number of LNs removed during PLND was significantly higher in the LD-group and the need of LD was significantly higher in the group that did not report an improvement regarding its LLL. This allows the interpretation that, the more LNs are removed, the more likely a severe course of the LLL (indicated by LD) 
gets and that it also is correlated to a more chronic state of LLL. This might especially affect patients who receive extended PLND. In this subpopulation the rate of postoperative lymphedema has been shown to be higher [13]. In a recent study among D'Amico intermediate and high-risk prostate cancer patients, Preisser et al. did not find significant difference in oncologic outcomes of patients in whom PLND was or was not performed [14]. Instead of performing extended PLND in all D'Amico high risk prostate cancer patients, the usage of a novel nomogram including mpMRI and MRI-targeted biopsy data could avoid up to $60 \%$ of PLNDs in this group at the cost of missing $1.6 \%$ of patients with lymph node invasion [15]. Among other effects (e.g., shorter operation time) this could reduce the rate of lymphedemas. This approach seems practicable because mpMRI becomes increasingly available. At the same time, the role of mpMRI in primary diagnostic is increasing [16]. A recent study by Ahdoot et al. showed combined biopsy was associated with fewer upgrades to unfavorable intermediate risk or worse when compared to MRI-targeted biopsy or systematic biopsy alone [17].

This survey's results showed radiotherapy following RP linked to a more chronic LLL in group comparison. Additionally, in simple linear regression, RT showed to influence results of total Lymph-ICFLL Score towards a more negative outcome. In the RADICALS-RT trial, Parker et al. detected that there was no difference regarding biochemical progression free survival between adjuvant and salvage radiotherapy in patients with at least one risk factor for prostate cancer progression after RP [18]. Because of the fact that the cohort receiving adjuvant radiotherapy showed more radiation therapy related side-effects and had an increased risk of urinary morbidity, they concluded that their results do not support routine administration of adjuvant radiotherapy after radical prostatectomy [18]. A change towards less adjuvant and more salvage radiotherapy could lead to less patients suffering from LLL and leave patients with less chronic states of LLL, especially since - next to surgical lymphatic vessel/node removal - radiation is the main cause for secondary lymphedemas [19].

Following the Clavien-Dindo classification for grades of severity of surgical complications and its 2015 extension by the Japan Clinical Oncology Group, LLLs that do not require intervention except for LD and CS are classified as grade I adverse event (AE) [20,21]. Medical management would classify as grade II AE and surgical intervention (e.g., lymphatic anastomosis) as grade III AE. Just 2/54 (3.7\%) patients classified as grade II LLL, whereas the rest classified as grade I LLL. The results of the PROM, measured via the Lymph-ICF-LL, especially the two domains physical function and mental function, on the other hand, prove that LLL entails a significant reduction in health-related quality of life and thus puts LLL in contrast to the low classification according to Clavien-Dindo. These impairments in health-related QoL are not sufficiently covered by the Clavien-Dindo classification and not well researched.

The results of the Lymph-ICF-LL show a significant impact of LLL on this populations' life. Especially mental and physical function were affected, which is also reflected in the significant difference of physical activity at the time of the survey between patients with an active and completely regressed LLL. Even though our data do not allow to conclude if patients are more active because LLL had regressed or if they have been more active all along, which might have been beneficial for regression of LLL, this finding is very important: Physical activity is linked to a better quality of life and health, shows favorable 
effects regarding reduction of chronic pain and reduces symptoms in anxiety and stress-related disorders as well as showing efficacy in treating symptoms of dementia $[22,23]$. Furthermore, it has been shown that it improves functional health and energy balance and reduces the risk of cardiovascular disease, stroke, diabetes, several cancers, depression and falls [24]. This is supported by the significant impact of the BMI on the Lymph-ICF-LL total score and findings that associate postdiagnosis obesity in prostate cancer patients with a higher not only cardiovascular disease-related but also all-cause mortality [25]. Thus, by affecting patients' physical activity, LLL most likely contributes to a vicious circle leading to a less active lifestyle, which paves the way for the aforementioned diseases. To help prevent that, education and awareness at the physicians' and patient's side as well as the routine integration of LLLinquiry and measurement in the postoperative follow-up is crucial. Especially, since the time difference between RP and LLL-diagnosis showed to be significantly longer in the group that eventually was in need of LD and significantly shorter in the group, in which the LLL partially or completely regressed.

The low percentage of patients receiving individually fitted CS for LLL (28/54 (51.9\%)) and the time gap between onset of LLL and receiving CSs ( $3.78 \pm 9.18$ months) as well as the high percentage of patients with an active LLL not using CSs (13/28 (46.4\%)) indicate a lack of awareness regarding this specific postoperative complication, which has been postulated before [5]. The fact that $24 \%(6 / 25)$ of patients, who received LD, did not receive CSs and only $27 \%$ (6/29) of patients, who did not receive LD, actually used CSs further contributes to that conclusion. Additionally, only 11/15 (73.3\%) patients with an active LLL receive LD. These findings are in contradiction to the fact that individually fitted CSs and LD are considered part of the standard of conservative lymphedema treatment and recommended by the German lymphedema guideline [26]. An increased awareness could be a step towards providing each patient with the recommended standard therapy. We do not consider the percentage of patients receiving CSs cost-related since they are covered by both the statutory and private health care insurance. Cost coverage also applies for LD.

\section{Limitations}

Limitations of this study include the cross-sectional design. LLL is not a steady state and the symptoms as well as the impairment of the subjective health and certain skills (e.g., physical and mental) can fluctuate over time. A prospective study design with patients, who all suffer from an active LLL, could provide further insights and reduce recall bias.

Another limitation is the non-homogeneous study population regarding when, where and by whom the patients have received their RP. This is due to the rarity of LLLs: In order to achieve a reasonable study size, we used a nation-wide email distribution list. At the same time this reflects the strength of the study, in terms that the results give a more representative insight how LLL is treated all over Germany and Lymph-ICF-LL could detect significant differences in a more heterogeneous population. Also, older patients or patients with lower incomes might not have been reached due to the web-based approach, which contributes to sample bias [27]. Furthermore, due to the web-based character using an email distribution list, we do not know how many patients were addressed, how many read the mail and which 
percentage of eligible patients took part in the survey and what the overall rate of LLL is. These details would have provided a more complete characterization of the complication LLL.

Since this is a patient-based survey, the collected data were self-reported and hence subjective, which is why the grade of LLL could not be assessed. An objective grading of the LLL would have provided more detailed insight and reduce a possible confounding effect by linking the therapy received to the grade of LLL.

\section{Conclusion}

To our knowledge, this is the first study characterizing the significant burden of LLL after RP with PLND on the patients' life, especially their mental and physical function. Postoperative onset of LLL is variable, which should be considered when assessing postoperative complications. This survey's findings indicate a gap between onset of LLL and provision of CSs as well as a high percentage of patients suffering from LLL, who not receive the recommended treatment, which contains CS and LLL. To diagnose and decrease disparity between diagnosis and treatment, in order to get more patients on the recommended basic therapy, an increase in public awareness and sufficient education and knowledge among urologists and patients undergoing RP is necessary.

\section{Abbreviations}

ADT $=$ Androgen deprivation therapy

CS = Individually fitted compression stockings

$\mathrm{ICHOM}=$ International consortium of health outcome measures

$\mathrm{IQR}=$ Interquartile Range

LE $=$ Lymphedema

$L L L=$ Lymphedema of the lower limb

$L D=$ Manual lymphatic drainage

Lymph-ICF-LL = Lymph-ICF-Questionnaire

$\mathrm{mpMRI}=$ Multiparametric magnetic resonance Imaging

PLND = Pelvic lymph node dissection

PROMs $=$ Patient-reported outcome measures

$\mathrm{RP}=$ Radical prostatectomy 
SF-12 $=$ Short Form 12 Health Survey

SD = Standard Deviation

\section{Declarations}

\section{Acknowledgements}

We gratefully acknowledge the men who generously gave their time for this study and thank the German Federal Association Prostate Cancer Self-Help e.V. (Bundesverband Prostatakrebs Selbsthilfe e.V.) for distributing the information and link to the survey. Furthermore, we want to thank Prof. Dr. Nele Devoogdt and Jana Allofs for the permission to use the Lymph-ICF-LL.

Funding: No funds, grants, or other support was received.

Conflicts of interest: M.N., L.S., M.L., C.M., N.W., P.N. and J.H have nothing to disclose. Dr. Wessels reports grants from Federal Ministry of Health, outside the submitted work.

\section{Availability of data and material: N/A}

Code availability: N/A

Authors' contributions: Conceptualization: Jost von Hardenberg, Manuel Neuberger; Methodology: Manuel Neuberger, Jost von Hardenberg, Laura Schmidt, Miriam Linke, Carina Müller, Frederik Wessels; Formal analysis and investigation: Manuel Neuberger, Laura Schmidt, Niklas Westhoff, Jost von Hardenberg, Frederik Wessels; Writing - original draft preparation: Manuel Neuberger, Jost von Hardenberg; Writing review and editing: Laura Schmidt, Frederik Wessels, Miriam Linke, Carina Müller, Niklas Westhoff, Philipp Nuhn; Funding acquisition: not applicable; Resources: not applicable; Supervision: Jost von Hardenberg, Manuel Neuberger

Ethics approval: Ethical approval was obtained from the University of Heidelberg's Ethics Committee II (Medical Faculty Mannheim, reference number 2021-517). The study was performed in accordance with the ethical standards as laid down in the 1964 Declaration of Helsinki and its later amendments or comparable ethical standards.

Consent to participate: Informed consent was obtained from all individual participants included in the study.

Consent to publish: N/A

\section{References}

1. Fletcher SA, von Landenberg N, Cole AP, Gild P, Choueiri TK, Lipsitz SR et al (2020) Contemporary national trends in prostate cancer risk profile at diagnosis. Prostate Cancer Prostatic Dis 23(1):81- 
87. Epub 2019/06/27

2. Mottet N, van den Bergh RCN, Briers E, Van den Broeck T, Cumberbatch MG, De Santis M et al (2021) EAU-EANM-ESTRO-ESUR-SIOG Guidelines on Prostate Cancer-2020 Update. Part 1: Screening, Diagnosis, and Local Treatment with Curative Intent. European urology 79(2):243-262. Epub 2020/11/12

3. Borkowetz A, Bruendl J, Drerup M, Herrmann J, Isbarn H, Beyer B (2018) Multicenter evaluation of guideline adherence for pelvic lymph node dissection in patients undergoing open retropubic vs. laparoscopic or robot assisted radical prostatectomy according to the recent German S3 guideline on prostate cancer. World J Urol 36(6):855-861. Epub 2018/02/11

4. Fu MR, Ridner SH, Hu SH, Stewart BR, Cormier JN, Armer JM (2013) Psychosocial impact of lymphedema: a systematic review of literature from 2004 to 2011. Psycho-oncology 22(7):14661484. Epub 2012/10/10

5. Keast DH, Despatis M, Allen JO, Brassard A (2015) Chronic oedema/lymphoedema: under-recognised and under-treated. International wound journal 12(3):328-333. Epub 2014/03/13

6. Martin NE, Massey L, Stowell C, Bangma C, Briganti A, Bill-Axelson A et al (2015) Defining a standard set of patient-centered outcomes for men with localized prostate cancer. European urology 67(3):460-467. Epub 2014/09/23

7. Coughlin GD, Yaxley JW, Chambers SK, Occhipinti S, Samaratunga H, Zajdlewicz L et al (2018) Robot-assisted laparoscopic prostatectomy versus open radical retropubic prostatectomy: 24-month outcomes from a randomised controlled study. The Lancet Oncology 19(8):1051-1060. Epub 2018/07/19

8. Pompe RS, Beyer B, Haese A, Preisser F, Michl U, Steuber T et al (2018) Postoperative complications of contemporary open and robot-assisted laparoscopic radical prostatectomy using standardised reporting systems. BJU Int 122(5):801-807. Epub 2018/05/05

9. Turner-Bowker D, Hogue SJ (2014) Short Form 12 Health Survey (SF-12). In: Michalos AC (ed) Encyclopedia of Quality of Life and Well-Being Research. Springer Netherlands, Dordrecht, pp 59545957

10. Bull FC, Al-Ansari SS, Biddle S, Borodulin K, Buman MP, Cardon G et al (2020) World Health Organization 2020 guidelines on physical activity and sedentary behaviour. Br J Sports Med 54(24):1451-1462. Epub 2020/11/27

11. Devoogdt N, De Groef A, Hendrickx A, Damstra R, Christiaansen A, Geraerts I et al (2014) Lymphoedema Functioning, Disability and Health Questionnaire for Lower Limb Lymphoedema (Lymph-ICF-LL): reliability and validity. Physical therapy 94(5):705-721. Epub 2014/01/15

12. Allofs J, Johnsen L, Devoogdt N, Stutterheim E, Hoppstädter W, Grüneberg C (2019) Interkulturelle Adaptation des Lymph-ICF-Fragebogens für Patienten mit Lymphödemen der unteren Gliedmaßen in Deutschland. physioscience 15(01):5-16

13. Clark T, Parekh DJ, Cookson MS, Chang SS, Smith ER Jr, Wells N et al (2003) Randomized prospective evaluation of extended versus limited lymph node dissection in patients with clinically 
localized prostate cancer. The Journal of urology 169(1):145-147; discussion 7-8. Epub 2002/12/13

14. Preisser F, van den Bergh RCN, Gandaglia G, Ost P, Surcel Cl, Sooriakumaran P et al (2020) Effect of Extended Pelvic Lymph Node Dissection on Oncologic Outcomes in Patients with D'Amico Intermediate and High Risk Prostate Cancer Treated with Radical Prostatectomy: A Multi-Institutional Study. The Journal of urology 203(2):338-343. Epub 2019/08/23

15. Gandaglia G, Ploussard G, Valerio M, Mattei A, Fiori C, Fossati N et al (2019) A Novel Nomogram to Identify Candidates for Extended Pelvic Lymph Node Dissection Among Patients with Clinically Localized Prostate Cancer Diagnosed with Magnetic Resonance Imaging-targeted and Systematic Biopsies. European urology 75(3):506-514. Epub 2018/10/22

16. Westhoff N, von Hardenberg J, Michel MS. [Intelligent early prostate cancer detection in 2021: more benefit than harm]. Der Urologe Ausg A. 2021. Epub 2021/04/22. Intelligente Prostatakarzinomfrüherkennung 2021 - mehr Nutzen als Schaden

17. Ahdoot M, Wilbur AR, Reese SE, Lebastchi AH, Mehralivand S, Gomella PT et al (2020) MRI-Targeted, Systematic, and Combined Biopsy for Prostate Cancer Diagnosis. N Engl J Med 382(10):917-928. Epub 2020/03/05

18. Parker CC, Clarke NW, Cook AD, Kynaston HG, Petersen PM, Catton C et al (2020) Timing of radiotherapy after radical prostatectomy (RADICALS-RT): a randomised, controlled phase 3 trial. Lancet 396(10260):1413-1421. Epub 2020/10/02

19. Huang J, Yu N, Wang X, Long X (2017) Incidence of lower limb lymphedema after vulvar cancer: A systematic review and meta-analysis. Medicine 96(46):e8722. Epub 2017/11/18

20. Dindo D, Demartines N, Clavien PA (2004) Classification of surgical complications: a new proposal with evaluation in a cohort of 6336 patients and results of a survey. Annals of surgery 240(2):205213. Epub 2004/07/27

21. Katayama H, Kurokawa Y, Nakamura K, Ito H, Kanemitsu Y, Masuda N et al (2016) Extended ClavienDindo classification of surgical complications: Japan Clinical Oncology Group postoperative complications criteria. Surg Today 46(6):668-685. Epub 2015/08/21

22. Ahlskog JE, Geda YE, Graff-Radford NR, Petersen RC. Physical exercise as a preventive or diseasemodifying treatment of dementia and brain aging. Mayo Clinic proceedings. 2011;86(9):876 - 84. Epub 2011/09/01

23. Penedo FJ, Dahn JR (2005) Exercise and well-being: a review of mental and physical health benefits associated with physical activity. Curr Opin Psychiatry 18(2):189-193. Epub 2006/04/28

24. Marzetti E, Calvani R, Tosato M, Cesari M, Di Bari M, Cherubini A et al (2017) Physical activity and exercise as countermeasures to physical frailty and sarcopenia. Aging clinical experimental research 29(1):35-42. Epub 2017/02/10

25. Troeschel AN, Hartman TJ, Jacobs EJ, Stevens VL, Gansler T, Flanders WD et al (2020) Postdiagnosis Body Mass Index, Weight Change, and Mortality From Prostate Cancer, Cardiovascular Disease, and 
All Causes Among Survivors of Nonmetastatic Prostate Cancer. Journal of clinical oncology: official journal of the American Society of Clinical Oncology 38(18):2018-2027. Epub 2020/04/07

26. Leitlinienprogramm Onkologie (Deutsche Krebsgesellschaft DK, AWMF). S2k Leitlinie Diagnostik und Therapie der Lymphödeme, AWMF Reg.-Nr. 058 - 001. 2017; Available from:

https://www.awmf.org/uploads/tx_szleitlinien/058-

0011_S2k_Diagnostik_und_Therapie_der_Lymphoedeme_2019-07.pdf (access date: 29.04.2021)

27. Ball HL (2019) Conducting Online Surveys. Journal of human lactation: official journal of International Lactation Consultant Association 35(3):413-417. Epub 2019/05/16

\section{Figures}

A

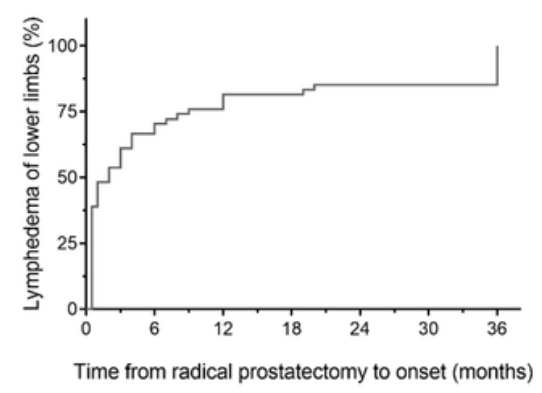

B

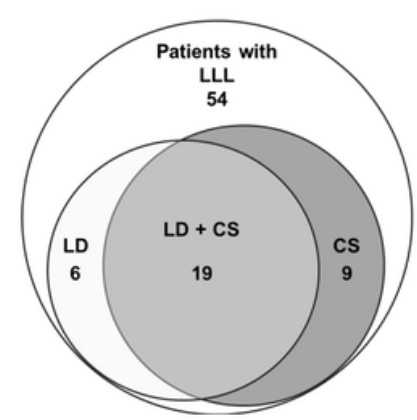

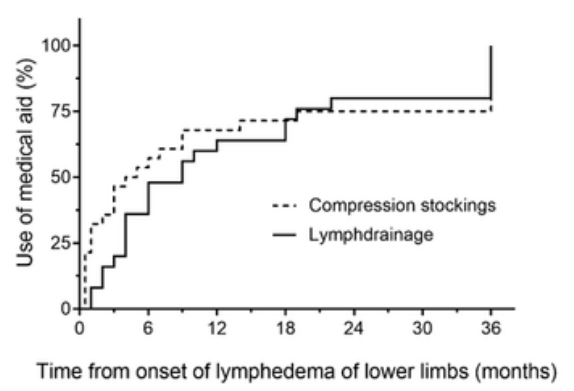

\section{Figure 1}

A Onset of lymphedema of the lower limbs (LLL) after radical prostatectomy for all 54 patients. B Quantitative Venn diagram showing the overlap of patients needing manual lymphatic drainage (LD) and compression stockings (CS) due to LLL in the entirety of LLL patients. C Onset of use of medical aids (CS and/or LD) of patients who reported the use of medical aids. 